\title{
Doppler shift extraction of wideband signal using spectrum scaling matching
}

\author{
ZHANG Fengzhen, LI Guijuan, ZHANG Zhaohui and $H U$ Chen
}

Dalian Scientific Test and Control Technology Institute, Science and Technology on Underwater Test and Control Laboratory, Dalian City, China

\begin{abstract}
Doppler shift is an important feature of moving targets. It can be used to extract target velocity, distance, track and other movement parameters. According to the problem of extracting Doppler shift for wideband signals with unstable line spectrum or no line spectrum, we proposed a Doppler shift extraction method for wideband signals based on spectral scaling matching. Firstly, a spectrum reference matrix corresponding to different relative Doppler shift is generated. Then, the matching degree of Doppler signal spectrum and reference matrix is measured by linear correlation coefficient. Finally, the Doppler shift of wideband signals is extracted through matching degree optimization. Simulation results show that the proposed method can extract the Doppler shift characteristics of wideband signals effectively.
\end{abstract}

\section{Introduction}

Doppler shift refers to the frequency difference between the target transmitting signal and the receiving signal of the observation point caused by the Doppler effect. Doppler shift involves target parameters about velocity, distance, trajectory, etc. It has an important application in many engineering problems. The line spectral Doppler shift of target radiated noise was used to estimate the distance and velocity parameters of underwater targets in $[1,2,3]$. Passive location was achieved by using the Doppler frequency shift characteristic of the aircraft's noise signal in $[4,5]$.

The key and prerequisite for estimation of target movement parameters were accurate and effectiveness of Doppler shift extraction. There are many methods for extracting Doppler frequency shift from line spectrum signal. Short Time Fourier Transform (STFT) can be directly used to extract Doppler shift by tracking the frequency variation of a line spectrum. There are other improved methods, such as a combined method of STFT and high resolution frequency estimation based on subspace rotation invariance technology, to improve the accuracy and resolution of frequency estimation [6].

However, for wideband signals with unstable line spectrum or no line spectrum, the methods based on line spectrum detection are ineffective to extract the Doppler shift. This causes that the above methods $[1,2,3,4]$ can't be applied to estimate the movement parameters of wideband signal target.

According to the above problem, the paper proposed a Doppler shift extraction method for wideband signals. This method takes advantage of the stretching effect principle of signal spectrum with Doppler shift. A reference matrix corresponding to a variety of relative
Doppler shift coefficients is established. Then the matching degree of Doppler frequency shift spectrum and reference matrix is measured by linear correlation coefficient to extract the Doppler frequency shift feature. The method solves the problem of extracting Doppler shift from wideband signals with unstable line spectrum or no line spectrum. So the target scope will be expanded about the movement parameters estimation based on Doppler shift.

\section{Principle and method}

The Doppler shift will cause the expansion of the signal spectrum. Similarly, the spectral sequences obtained from different Discrete Fourier Transform (DFT) point numbers correspond to different frequency resolutions. The Doppler shift of wideband signals can be extracted by establishing the relationship between the two.

\subsection{Signal spectrum expansion caused by Doppler shift}

According to the Doppler effect, the moving target signal received by the observation point will produce Doppler frequency shift. For frequency component $f_{i}$ of the target signal, the frequency offset is $\Delta f_{i}(t)$ :

$$
\Delta f_{i}(t)=\frac{v_{r}(t)}{c} f_{i}
$$

Where, $v_{r}(t)$ denotes the real-time velocity of the target relative to the observation point, $c$ denotes the signal propagation velocity in medium. For broadband signals, the Doppler shift of different frequency 
components is proportional to its frequency. The Doppler frequency shift is large for the high-frequency component and small for the low frequency component.

The ratio of the frequency shift to the corresponding frequency can be defined as the relative Doppler coefficient without considering Doppler frequency shift at the specific frequency of the signal. It can be expressed as $a(t)$ :

$$
a(t)=\frac{\Delta f_{i}(t)}{f_{i}}=\frac{v_{r}(t)}{c}
$$

The $a(t)$ is the ratio between the velocity $v_{r}(t)$ of the target relative to the observation point and the signal propagation velocity $\mathrm{c}$ in the medium actually. It reflects the frequency shift ratio of broadband signal at any frequency. For $a>0$, the target is moving closer to the observation point, and the signal frequency is shifted up. Conversely, $a<0$ indicates that the target is away from the observation point, and the signal frequency is shifted downward.

Fig. 1 is the spectrum of a wideband signal after Doppler shift with a relative Doppler shift coefficient $a=0.02$. It is shown that the spectrum of Doppler shift signal has a significant extension effect on the structure compared with the original.

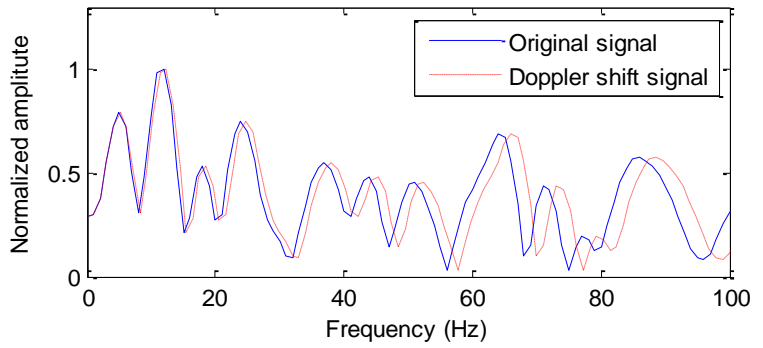

Fig 1. Spectrum expansion of Doppler shift signal.

\subsection{Spectrum expansion caused by different DFT points}

The DFT series of time domain signals $s(n)$ is defined [7] as:

$$
S(k)=\sum_{m=1}^{N-1} s(n) e^{-j \frac{2 \pi}{N} k m},(m=0,1, \ldots, N-1)
$$

For the time series of signal with sampling rate $F_{s}$, the frequency resolution of $M$ point DFT spectrum sequence is $F_{s} / M$. So, the position of line spectrum $f_{i}$ in the frequency sequence is $M f_{i} / F_{s}$.

Therefore, for discrete time series, there is a kind of "expand or contract" relationship between the frequency sequences with different DFT points. By increasing the point of DFT, the frequency spectrum of the signal has a kind of stretching effect compared with the original. The frequency spectrum of the signal has a kind of compression effect compared with the original spectrum with DFT points reduced.

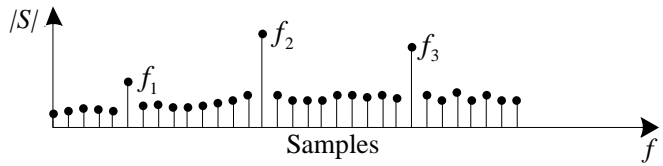

(a)

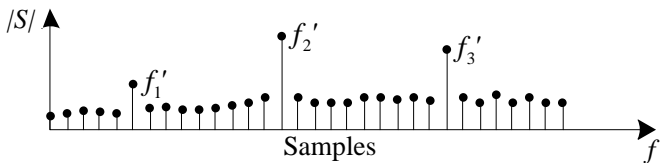

(b)

Fig 2. Spectrum expansion diagram of different DFT points.

Assume the spectrum sequence of signal is shown in Fig. 2(a). When the sequence is extended to the situation shown in Fig. 2(b), its frequency increases with directly proportional to its frequency. Without loss of generality, the frequency variation $f_{i}^{\prime}$ of spectral line $f_{i}$ is as follows:

$$
f_{i}^{\prime}=f_{i}+\Delta f_{i}=f_{i}\left(1+\frac{\Delta f_{i}}{f_{i}}\right)
$$

Therefore, in order to obtain the frequency expansion of Fig. 2(b) relative to Fig. 2(a), the method of reducing the DFT points can be used to compress the extended spectrum, and match with the original spectrum sequence.

It's easy to know, when the coordinate of the extended spectrum line $f_{i}^{\prime}$ is compressed to the frequency $f_{i}$, the best match between the spectrum and the original spectrum can be made. In this case,

$$
\frac{N f_{i}}{F_{s}}=\frac{(N+\Delta N) f_{i}^{\prime}}{F_{s}}
$$

Combined (4) and (5), it can be obtained:

$$
\frac{-\Delta N}{N+\Delta N}=\frac{\Delta f_{i}}{f_{i}}
$$

Therefore, if the appropriate number of DFT points is chosen to match the signal spectrum scalability of the signal frequency shift, the signal Doppler shift coefficient can be obtained by matching the signal spectrum expansion of the signal frequency shift.

$$
a=-\frac{\Delta N}{N+\Delta N}
$$

For the signal with the same sampling rate $F_{s}$ and signal analysis window length $N$, the resolution $r_{1}$ of Doppler shift coefficient of the method is

$$
r_{1} \leq \frac{1}{N+1}
$$

When using the conventional line spectrum analysis and tracking method to extract the Doppler shift coefficient, the accuracy is related to the frequency of the line spectrum. The resolution is the best when the 
line spectrum is located at the upper limit $F_{s} / 2$ of effective frequency. Therefore, the Doppler frequency shift coefficient extracting resolution $r_{2}$ of the method is

$$
r_{2} \geq \frac{F_{s}}{N} / \frac{F_{s}}{2}=\frac{2}{N}
$$

Consequently,

$$
r_{2}>2 r_{1}
$$

It can be seen that the resolution of the Doppler shift coefficient extracted by the proposed method is at least 2 times higher than that by the traditional line spectrum analysis and tracking method.

\subsection{Extraction method and processes}

According to the above principle of Doppler shift coefficient extraction, the steps of extracting the Doppler frequency shift characteristics of a portion of signal $s(n)$ are as follows:

(1) Firstly, select a segment of data in the signal as a reference signal. Fast Fourier transform (FFT) with $N, N \pm 1, N \pm 2, \ldots N \pm \Delta N$ points are conducted to the reference signal respectively. And the DFT series are combined into the spectrum expansion reference matrix $\mathbf{F}_{\mathbf{0}}$ in which different spectrum sequences have different scaling and corresponding different Doppler frequency shift coefficients. The selection of $\Delta N$ should ensure that the maximum Doppler shift of the signal can be analyzed. And the reference signal segment usually selects the front of the signal, especially when it is used in real time.

(2) Divide the signal into segments, process them by $\mathrm{N}$ point $\mathrm{FFT}$ and obtained the spectrum sequence $\mathrm{F}$. Then, use linear correlation coefficient to measure the matching degree between $F$ and each spectral sequence $F_{0 i}$ of the spectrum scaling reference matrix $\mathbf{F}_{\mathbf{0}}$.

$$
R_{i}=\frac{\operatorname{cov}\left(F, F_{0 i}\right)}{\sqrt{\operatorname{var}(F) \cdot \operatorname{var}\left(F_{0 i}\right)}}
$$

Where, $\operatorname{cov}\left(F, F_{0 i}\right)$ is the covariance of $F$ and $F_{0 i}$, $\operatorname{var}(F)$ and $\operatorname{var}\left(F_{0 i}\right)$ are the variance of $F$ and $F_{0 i}$, respectively.

(3) Find the FFT points $M$ corresponding to the maximum value of the linear correlation coefficient sequence R. Then, the Doppler shift coefficient of the signal relative to the reference signal is

$$
a=\frac{N}{M}-1
$$

According to the above steps, the Doppler frequency shift coefficients of signals can be extracted by processing the segmented signals successively.

\section{Simulation signal processing}

The parameters of the signal simulation are set as follows. Suppose that in a two-dimensional coordinate system, a broadband signal target moves at a speed of $v$ along the straight line parallel to the $\mathrm{x}$-axis. The nearest vertical distance between the observation point $R$ and the sound source trajectory is $d$. The propagation velocity of the signal in the medium is $c$. The location of the target and observation point is shown in Fig. 3.

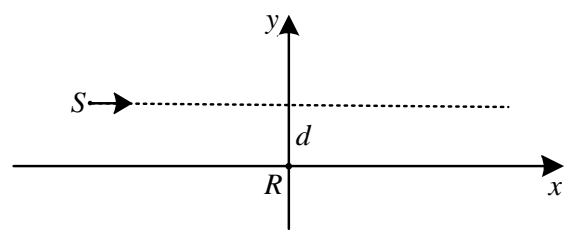

Fig 3. Diagram of the moving target and the observation position.

Suppose that the broadband signal source radiate steady broadband random noise into the medium. The noise signal is obtained by bandpass filtering of white Gauss noise. The frequency band of the signal is $1 \mathrm{kHz} \sim 3 \mathrm{kHz}$. The sampling frequency is $12 \mathrm{kHz}$. The spectrum of the simulation signal is shown in Fig. 4. There is no line spectrum component in the signal.

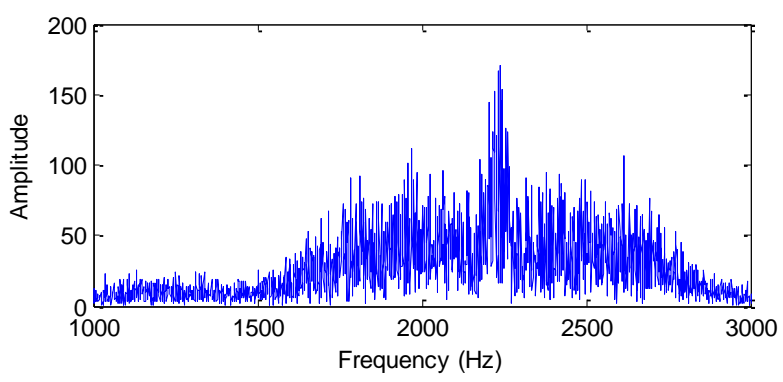

Fig 4. Signal spectrum diagram.

The signal received by the observation point $R$ is the Doppler shifted signal. In the 20 s time before and after the closest source location, the time-frequency spectrum of the simulation signal is shown in Fig. 5. It can be seen that it has obvious spectrum scaling effect at different time. The signal spectrum moves up when the source is closing to the observation point. And the signal spectrum moves down when the sound source away from the observation point.

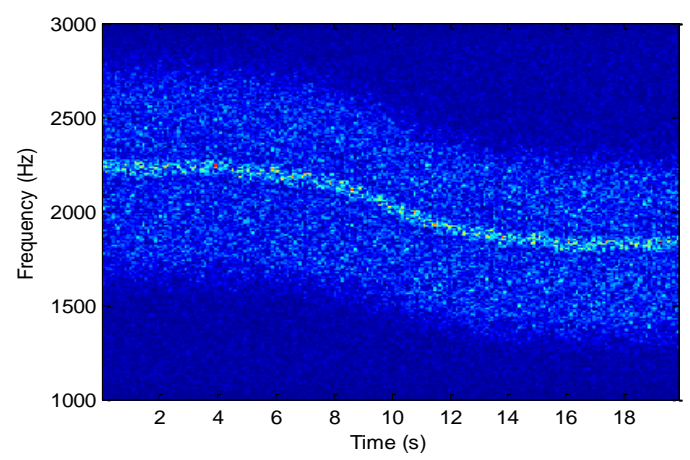

Fig5. Time-frequency spectrum of the signal. 
According to the method proposed in this paper, the wideband signal with Doppler shift is processed. Firstly, the reference matrix should be set up, and then the scaling and matching of the frequency spectrum of the segmented signal and the spectrum of the reference matrix should be measured to calculate the Doppler shift coefficient of the signals of each segment.

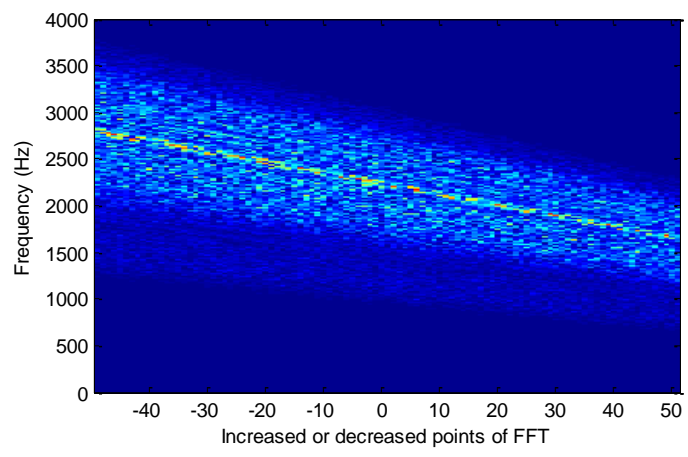

Fig 6. Image of spectrum matching reference matrix.

For band limited signals, when constructing reference matrix, only the spectrum sequence values in the effective frequency band should be reserved, but enough margin should be left out. The simulation signal is processed with the window width of 1 second. The spectrum sequence values below $4 \mathrm{kHz}$ is retained to establish the reference matrix. The reference matrix is represented as the image shown in Fig. 6. For the spectrum of segmented signals, the same number of points in the reference matrix is retained, in order to match the frequency spectrum easily.

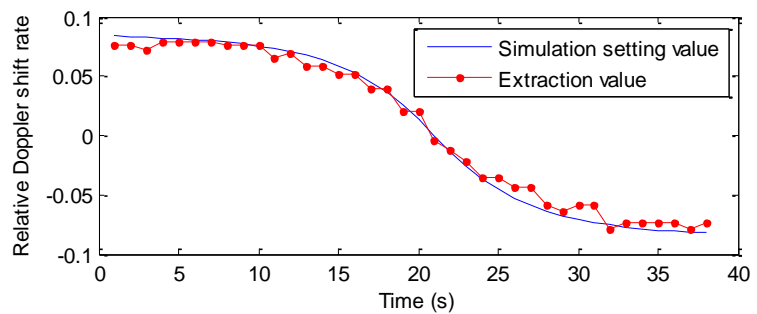

Fig 7. Relative Doppler shift coefficient extraction results.

According to the proposed method, the Doppler frequency shift coefficient extracted from the simulated signal is shown in Fig. 7. It is shown that the extraction results of the signal Doppler coefficient have good accuracy, the maximum absolute error is 0.015 , the minimum absolute error is 0.01 . The error is very small compared with the simulation set value.

\section{Conclusion}

In this paper, an extraction method of signal Doppler shift is proposed for the broadband signal with unstable line spectrum or no line spectrum. The method uses the consistency of signal spectrum expansion caused by Doppler shift and spectrum expansion caused by different DFT points. The relationship between the spectral analysis points and the Doppler shift coefficient is established. By means of the reference matrix corresponding to different Doppler frequency shift coefficients, the matching degree between the signal spectrum and the reference matrix can be measured by linear correlation coefficient. Then, the characteristics of the relative Doppler frequency shift coefficient are extracted by optimization. The proposed method has higher accuracy of Doppler shift analysis than the traditional method based on short time Fourier transform. Doppler frequency shift signal of moving target was simulated and processed. Results show that the method can extract the Doppler shift characteristics of the broadband signal without line spectrum with good precision. This method will expand the target application scope of target movement parameters estimation based on Doppler shift and has practical application value.

\section{References}

1. WU Guoqing, MA Li, ACTA ACUSTICA, 31, 14 (2006)

2. XU Lingji, YANG Yixin, Journal of Harbin Engineering University, 34, 1090 (2013)

3. GAO Wei, CHEN Chuan, Technical Acoustics, 29, 374 (2010).

4. David C. Reid, Abdelhak M. Zoubir, Boualem Boashash. J. Acoust. Soc. Am., 102, 207 (1997)

5. David R. Dall'Osto, Peter H. Dahl, J. Acoust. Soc. Am., 127, 1771 (2010)

6. XU Ling-ji, YANG Yi-xin, TIAN Feng, Technical Acoustics, 30, 523 (2011) 